\title{
AN UNCERTAINTY PRINCIPLE FOR THE DUNKL TRANSFORM
}

\author{
MARGIT RÖSLER
}

This note presents an analogue of the classical Heisenberg-Weyl uncertainty principle for the Dunkl transform on $\mathbb{R}^{N}$. Its proof is based on expansions with respect to generalised Hermite functions.

\section{INTRODUCTION}

The Dunkl transform is an integral transform on $\mathbb{R}^{N}$ which generalises the classical Fourier transform. On suitable function spaces, it establishes a natural correspondence between the action of multiplication operators on one hand and so-called Dunkl operators on the other. These are differential-difference operators, generalising the usual partial derivatives, which are associated with a finite reflection group on some Euclidean space. They play, for example, a useful role in the algebraic description of exactly solvable quantum many body systems of Calogero-Moser-Sutherland type; among the broad literature in this context, we refer to [1], [9], and [11]. In his paper [8], de Jeu proved a quite general uncertainty principle for integral operators with bounded kernel which applies to the Dunkl transform; this result has the form of an $\varepsilon-\delta$-concentration principle as first stated in [4] for the Fourier transform. Analogues of the classical variance-based Weyl-Heisenberg uncertainty principle for the Dunkl transform have up to now only been given in the one-dimensional case ([14] and [15]). It is the aim of this note to present an extension to general Dunkl transforms in arbitrary dimensions. Our setting, which is described in more detail in section 2, is as follows: Let $R$ be a finite (reduced) root system on $\mathbb{R}^{N}$ and $k: R \rightarrow[0, \infty]$ a nonnegative multiplicity function on $R$. Let $w_{k}$ denote the weight function

$$
w_{k}(x)=\prod_{\alpha \in R}|\langle\alpha, x\rangle|^{k(\alpha)}
$$

on $\mathbb{R}^{N}$, where $\langle.,$.$\rangle is the Euclidean scalar product on \mathbb{R}^{N}$, and put $\gamma:=\sum_{\alpha \in R} k(\alpha) / 2$. We shall prove the following uncertainty principle for the associated Dunkl transform $f \mapsto \widehat{f}^{k}$ on $L^{2}\left(\mathbb{R}^{N}, w_{k}(x) d x\right)$ :

Received 26th August, 1998

I am indebted to Richard Askey, who stimulated this work by bringing C. Roosenraad's thesis [15] to my attention.

Copyright Clearance Centre, Inc. Serial-fee code: 0004-9729/99 $\$ A 2.00+0.00$. 
THEOREM 1.1. Let $f \in L^{2}\left(\mathbb{R}^{N}, w_{k}(x) d x\right)$. Then

$$
\||x| f\|_{2, w_{k}} \cdot\left\||\xi| \hat{f}^{k}\right\|_{2, w_{k}} \geqslant(\gamma+N / 2) \cdot\|f\|_{2, w_{k}} .
$$

Moreover, equality holds if and only if $f(x)=c e^{-d|x|^{2}}$ for some constants $c \in \mathbb{C}$ and $d>0$.

If the multiplicity function $k$ is identically 0 , then the corresponding Dunkl transform coincides with the usual Fourier transform (independently of the underlying root system), and the above result coincides with the classical Weyl-Heisenberg inequality on $L^{2}\left(\mathbb{R}^{N}\right)$.

Our proof of Theorem 1.1 is based on expansions in terms of generalised Hermite functions, which were introduced in [12]. This generalises a well-known method for the (one-dimensional) classical situation, see for example, [2]. Our method needs not much more effort than in the classical case, but requires a zero-centred situation. This restriction cannot easily be removed. For the one-dimensional case, the result of Theorem 1.1 was already obtained in [15], by a very similar method. In contrast, the version given in [14] is uncentred. It is based on commutator methods which become difficult to handle in higher dimensions. However, the lower bound in [14] is not uniform, and coincides with the one above for even functions only.

\section{DUNKL OPERATORS AND THE DUNKL TRANSFORM}

In this section, we collect some basic facts from Dunkl's theory which will be needed later on. General references here are $[7,5,6]$.

For $\alpha \in \mathbb{R}^{N} \backslash\{0\}$ we denote by $\sigma_{\alpha}$ the reflection in the hyperplane orthogonal to $\alpha$, given by $\sigma_{\alpha}(x)=x-\left(2\langle\alpha, x\rangle /|\alpha|^{2}\right) \alpha$. Let $R$ be a (reduced) root system in $\mathbb{R}^{N}$, that is, a finite subset of $\mathbb{R}^{N} \backslash\{0\}$ with $R \cap \mathbb{R} \cdot \alpha=\{ \pm \alpha\}$ and $\sigma_{\alpha}(R)=R$ for all $\alpha \in R$. We assume that the root system $R$ is normalised, that is, $|\alpha|^{2}=2$ for all $\alpha \in R$. The reflections $\sigma_{\alpha}, \alpha \in R$ generate a finite group $G$, the reflection group associated with $R$. A function $k: R \rightarrow \mathbb{C}$ is called a multiplicity function on $R$ if it is invariant under the natural action of $G$ on $R$. Now fix a reflection group $G$ on $\mathbb{R}^{N}$ and a multiplicity function $k \geqslant 0$ on its root system $R$. The Dunkl operators $T_{i}(i=1, \ldots, N)$ on $\mathbb{R}^{N}$ associated with $G$ and $k$ are defined by

$$
T_{i} f(x):=\partial_{i} f(x)+\frac{1}{2} \sum_{\alpha \in R} k(\alpha) \alpha_{i} \cdot \frac{f(x)-f\left(\sigma_{\alpha} x\right)}{\langle\alpha, x\rangle}, \quad f \in C^{1}\left(\mathbb{R}^{N}\right)
$$

here $\partial_{i}$ denotes the $i$-th partial derivative. In the case $k=0$, the $T_{i}$ reduce to the usual partial derivatives. In this paper, we assume that all values of $k$ are nonnegative, for short, $k \geqslant 0$. The most important basic properties of the operators $T_{i}$ are as follows: Let $\mathcal{P}=\mathbb{C}\left[x_{1}, \ldots, x_{N}\right]$ denote the algebra of polynomial functions on $\mathbb{R}^{N}$ and $\mathcal{P}_{n}\left(n \in \mathbb{Z}_{+}=\right.$ $\{0,1, \ldots\})$ the subspace of homogeneous polynomials of degree $n$. Then 
(1.1) Each $T_{i}$ is homogeneous of degree -1 on $\mathcal{P}$, that is, $T_{i} p \in \mathcal{P}_{n-1}$ for $p \in \mathcal{P}_{n}$.

(1.2) The set $\left\{T_{i}, i=1, \ldots, N\right\}$ generates a commutative algebra of differentialdifference operators on $\mathcal{P}$.

For a polynomial $p \in \mathcal{P}$, we denote by $p(T)$ the linear operator derived from $p(x)$ by replacing $x_{i}$ by $T_{i}$. In particular, the generalised Laplacian is defined by $\Delta_{k}:=p(T)$ with $p(x)=|x|^{2}$. Note that $\Delta_{k}$ is homogeneous of degree -2 , and hence for each $c \in \mathbb{C}$, the exponential $e^{c \Delta_{k}}$ is a well-defined linear operator on $\mathcal{P}$ with inverse $e^{-c \Delta_{k}}$.

The solution of the joint eigenfunction problem for the Dunkl operators $\left\{T_{i}, i=\right.$ $1, \ldots, N\}$ is given by the Dunkl kernel $K_{G}$ on $\mathbb{R}^{N} \times \mathbb{R}^{N}$ : for each fixed $y \in \mathbb{R}^{N}$, the function $x \mapsto K_{G}(x, y)$ is characterised as the unique solution of the system $T_{i} f=$ $y_{i} f(i=1, \ldots, N)$ with $f(0)=1$; see [10]. The kernel $K_{G}(x, y)$ is symmetric in its arguments and has a unique holomorphic extension to $\mathbb{C}^{N} \times \mathbb{C}^{N}$. It satisfies $K_{G}(z, 0)=1$ and $K_{G}(\lambda z, w)=K_{G}(z, \lambda w)$ for all $z, w \in \mathbb{C}^{N}$ and all $\lambda \in \mathbb{C}$. Moreover, the function $x \mapsto K_{G}(i x, y)$. ( $y \in \mathbb{R}^{N}$ fixed) is positive definite on $\mathbb{R}^{N}$. See [13]. In particular, $\left|K_{G}(i x, y)\right| \leqslant 1$ for all $x, y \in \mathbb{R}^{N}$.

The Dunkl transform associated with $G$ and $k$ is given by

$$
\begin{aligned}
\hat{\imath}^{k} & : L^{1}\left(\mathbb{R}^{N}, w_{k}(x) d x\right) \rightarrow C_{b}\left(\mathbb{R}^{N}\right) \\
\hat{f}^{k}(\xi) & :=2^{-\gamma-N / 2} c_{k} \int_{\mathbb{R}^{N}} f(x) K_{G}(-i \xi, x) w_{k}(x) d x\left(\xi \in \mathbb{R}^{N}\right),
\end{aligned}
$$

with the Mehta-type constant

$$
c_{k}:=\left(\int_{\mathbb{R}^{N}} e^{-|x|^{2}} w_{k}(x) d x\right)^{-1}
$$

This transformation has many properties analogous to the Fourier transform on $\mathbb{R}^{N}$, among which we shall in particular need the following:

PROPOSITION 2.1. [7]

(1) The Dunkl transform $f \rightarrow \hat{f}^{k}$ is a homeomorphism of the Schwartz space $\mathcal{S}\left(\mathbb{R}^{N}\right)$ of rapidly decreasing functions on $\mathbb{R}^{N}$.

(2) ${\widehat{T_{j} f}}^{k}(\xi)=i \xi_{j} \hat{f}^{k}$ for all $f \in \mathcal{S}\left(\mathbb{R}^{N}\right)$ and $j=1, \ldots, N$.

(3) (Plancherel theorem) The Dunkl transform has a unique extension to an isometric isomorphism of $L^{2}\left(\mathbb{R}^{N}, w_{k}(x) d x\right)$, which is again denoted by $f \rightarrow$ $\widehat{f}^{k}$.

ExAmPLES 2.2. (1) If $k=0$, then $K_{G}(z, w)=e^{(z, w)}$ for all $z, w \in \mathbb{C}^{N}$. Here the Dunkl transform is the usual Fourier transform on $\mathbb{R}^{N}$.

(2) If $N=1$ and $G=\mathbb{Z}_{2}$, sending $x \in \mathbb{R}$ to $-x$, then the multiplicity function is a single parameter $k \geqslant 0$, and the Dunkl kernel is given by

$$
K_{\mathbf{Z}_{2}}(z, w)=j_{k-1 / 2}(i z w)+\frac{z w}{2 k+1} j_{k+1 / 2}(i z w) \quad(z, w \in \mathbb{C})
$$


where for $\alpha \geqslant-1 / 2, j_{\alpha}$ is the normalised spherical Bessel function

$$
j_{\alpha}(z)=2^{\alpha} \Gamma(\alpha+1) \frac{J_{\alpha}(z)}{z^{\alpha}}=\Gamma(\alpha+1) \cdot \sum_{n=0}^{\infty} \frac{(-1)^{n}(z / 2)^{2 n}}{n ! \Gamma(n+\alpha+1)}
$$

The corresponding Dunkl transform coincides with the Fourier transform on a certain (signed) hypergroup structure on $\mathbb{R}$; for details see [14] and the literature cited there.

\section{Generalised Hermite functions}

Let $G$ be a finite reflection group on $\mathbb{R}^{N}$ and $k \geqslant 0$ a fixed multiplicity function on its root system $R$. In [12] we introduced complete systems of orthogonal polynomials with respect to the weight function $w_{k}(x) e^{-|x|^{2}}$ on $\mathbb{R}^{N}$, called generalised Hermite polynomials. The key to their definition is the following bilinear form on $\mathcal{P}$, which was introduced in [6]:

$$
[p, q]_{k}:=(p(T) q)(0) \text { for } p, q \in \mathcal{P}
$$

The homogeneity of the Dunkl operators implies that $\mathcal{P}_{n} \perp \mathcal{P}_{m}$ for $n \neq m$. Moreover, if $p, q \in \mathcal{P}_{n}$, then

$$
[p, q]_{k}=2^{n} c_{k} \int_{\mathbf{R}^{N}} e^{-\Delta_{k} / 4} p(x) e^{-\Delta_{k} / 4} q(x) e^{-|x|^{2}} w_{k}(x) d x
$$

This is obtained from Theorem 3.10 of [6] by rescaling, see [12, Lemma 2.1]. So in particular, $[., .]_{k}$ is a scalar product on the vector space $\mathcal{P}_{\mathbb{R}}=\mathbb{R}\left[x_{1}, \ldots, x_{N}\right]$.

Now let $\left\{\varphi_{\nu}, \nu \in \mathbb{Z}_{+}^{N}\right\}$ be an (arbitrary) orthonormal basis of $\mathcal{P}_{\mathbb{R}}$ with respect to $[.,]_{k}$ such that $\varphi_{\nu} \in \mathcal{P}_{|\nu|}$. (For details concerning the construction and canonical choices of such a basis, we refer to [12]). Then the generalised Hermite polynomials $\left\{H_{\nu}, \nu \in \mathbb{Z}_{+}^{N}\right\}$ and the (normalised) generalised Hermite functions $\left\{h_{\nu}, \nu \in \mathbb{Z}_{+}^{N}\right\}$ associated with $G, k$ and $\left\{\varphi_{\nu}\right\}$ are defined by

$$
H_{\nu}(x):=2^{|\nu|} e^{-\Delta_{k} / 4} \varphi_{\nu}(x) \quad \text { and } \quad h_{\nu}(x):=\sqrt{c_{k}} 2^{-|\nu| / 2} e^{-|x|^{2} / 2} H_{\nu}(x) \quad\left(x \in \mathbb{R}^{N}\right)
$$

Note that $H_{\nu}$ is a polynomial of degree $|\nu|$, with real coefficients. This implies $(3 N$ term) recurrencies of the following form: For $\nu \in \mathbb{Z}_{+}^{N}$, let $I_{\nu}=\left\{\mu \in \mathbb{Z}_{+}^{N}:\|\mu|-| \nu\| \leqslant 1\right\}$. Then

$$
x_{j} H_{\nu}=\sum_{\mu \in I_{\nu}} c_{\nu, \mu}^{j} H_{\mu} \quad \text { and } \quad x_{j} h_{\nu}=\sum_{\mu \in I_{\nu}} c_{\nu, \mu}^{j} h_{\mu} \quad \text { for } j=1, \ldots, N
$$

with coefficients $c_{\nu, \mu}^{j} \in \mathbb{R}$. In general, there are many possible choices of generalised Hermite systems. However, in the one-dimensional case $N=1$ (with fixed parameter $k \geqslant 0)$, the basis $\left\{\varphi_{n}, n \in \mathbb{Z}_{+}\right\}$is uniquely determined. The associated generalised Hermite polynomials are orthogonal with respect to the weight function $|x|^{2 k} e^{-|x|^{2}}$ on $\mathbb{R}$ 
and can be written explicitly in terms of Laguerre polynomials; for details, see [12] or [3, Chapter V].

We collect some further properties of the generalised Hermite functions $\left\{h_{\nu}, \nu \in \mathbb{Z}_{+}^{N}\right\}$ which will be essential for the proof of Theorem 1.1.

LEMMA 3.1. [12]

(1) $\left\{h_{\nu}, \nu \in \mathbb{Z}_{+}^{N}\right\}$ is an orthonormal basis of $L^{2}\left(\mathbb{R}^{N}, w_{k}(x) d x\right)$.

(2) The $h_{\nu}$ are eigenfunctions of the Dunkl transform on $L^{2}\left(\mathbb{R}^{N}, w_{k}(x) d x\right)$, with $\widehat{h}_{\nu}^{k}=(-i)^{|\nu|} h_{\nu}$.

(3) The $h_{\nu}$ satisfy $\left(|x|^{2}-\Delta_{k}\right) h_{\nu}=(2|\nu|+2 \gamma+N) h_{\nu}$.

\section{Proof of the UNCERTAINTY PRINCIPLE}

From now on, $\left\{h_{\nu}, \nu \in \mathbb{Z}_{+}^{N}\right\}$ is an arbitrary fixed system of generalised Hermite functions associated with $G$ and $k \geqslant 0$. We shall need the dual counterparts of the recurrences (3.2):

$$
T_{j} h_{\nu}=\sum_{\mu \in I_{\nu}} i^{1-|\nu|+|\mu|} c_{\nu, \mu}^{j} h_{\mu} \quad\left(j=1, \ldots, N, \nu \in \mathbb{Z}_{+}^{N} .\right)
$$

These are easily obtained from (3.2) by use of Proposition 2.1.(2) and Lemma 3.1.(2).

We write $\langle.,\rangle_{k}$ for the scalar product in $L^{2}\left(\mathbb{R}^{N}, w_{k}(x) d x\right)$. The main part in the proof of Theorem 1.1 is the following Parseval-type identity.

Lemma 4.1. Let $f \in L^{2}\left(\mathbb{R}^{N}, w_{k}(x) d x\right)$. Then

$$
\int_{\mathbb{R}^{N}}|x|^{2}\left(|f(x)|^{2}+\left|\hat{f}^{k}(x)\right|^{2}\right) w_{k}(x) d x=\sum_{\nu \in \mathbf{Z}_{+}^{N}}(2|\nu|+2 \gamma+N) \cdot\left|\left\langle f, h_{\nu}\right\rangle_{k}\right|^{2} .
$$

Proof: Fix $j \in\{1, \ldots, N\}$. In view of Lemma 3.1.(1), we can write

$$
\int_{\mathbb{R}^{N}}\left|x_{j}\right|^{2}|f(x)|^{2} w_{k}(x) d x=\sum_{\nu \in \mathbf{Z}_{+}^{N}}\left|\left\langle x_{j} f, h_{\nu}\right\rangle_{k}\right|^{2}=\sum_{\nu \in \mathbf{Z}_{+}^{N}}\left|\left\langle f, x_{j} h_{\nu}\right\rangle_{k}\right|^{2} .
$$

By use of (3.2), this becomes

$$
\sum_{\nu \in \mathbf{Z}_{+}^{N}} \sum_{\mu, \rho \in I_{\nu}} c_{\nu, \mu}^{j} c_{\nu, \rho}^{j} \cdot\left\langle f, h_{\mu}\right\rangle_{k} \overline{\left\langle f, h_{\rho}\right\rangle_{k}}=\sum_{\mu, \rho \in \mathbf{Z}_{+}^{N}}\left(\sum_{\nu \in I_{\mu} \cap I_{\rho}} c_{\nu, \mu}^{j} c_{\nu, \rho}^{j}\right)\left\langle f, h_{\mu}\right\rangle_{k} \overline{\left\langle f, h_{\rho}\right\rangle_{k}} .
$$

Here the last equality is justified by the facts that the involved index sets $I_{\nu}$ are finite, and that $\mu \in I_{\nu} \Longleftrightarrow \nu \in I_{\mu}$ holds for all $\nu, \mu \in \mathbb{Z}_{+}^{N}$. Exploiting Lemma 3.1.(2), Proposition 2.1.(2) and the Parseval identity for the Dunkl transform, one further obtains

$$
\begin{aligned}
\int_{\mathbf{R}^{N}}\left|x_{j}\right|^{2}\left|\widehat{f}^{k}(x)\right|^{2} w_{k}(x) d x=\sum_{\nu \in \mathbf{Z}_{+}^{N}}\left|\left\langle x_{j} \widehat{f}^{k}, h_{\nu}\right\rangle_{k}\right|^{2} & =\sum_{\nu \in \mathbf{Z}_{+}^{N}}\left|\left\langle\widehat{f}^{k}, x_{j} \widehat{h}_{\nu}^{k}\right\rangle_{k}\right|^{2} \\
& =\sum_{\nu \in \mathbf{Z}_{+}^{N}}\left|\left\langle f, T_{j} h_{\nu}\right\rangle_{k}\right|^{2}
\end{aligned}
$$


With the recurrence $(4.1)$, this becomes

$$
\begin{aligned}
\sum_{\nu \in \mathbf{Z}_{+}^{N}} \sum_{\mu, \rho \in I_{\nu}} i^{|\nu|-|\mu|-1} c_{\nu, \mu}^{j} \cdot i^{1-|\nu|+|\rho|} c_{\nu, \rho}^{j} \cdot\left\langle f, h_{\mu}\right\rangle_{k} \overline{\left\langle f, h_{\rho}\right\rangle_{k}} & \\
& =\sum_{\mu, \rho \in \mathbf{Z}_{+}^{N}}\left(\sum_{\nu \in I_{\mu} \cap I_{\rho}} c_{\nu, \mu}^{j} c_{\nu, \rho}^{j}\right) i^{|\rho|-|\mu|} \cdot\left\langle f, h_{\mu}\right\rangle_{k} \overline{\left\langle f, h_{\rho}\right\rangle_{k}} .
\end{aligned}
$$

Combining the previous results, we arrive at

$$
\int_{\mathbb{R}^{N}}|x|^{2}\left(|f(x)|^{2}+\left|\hat{f}^{k}(x)\right|^{2}\right) w_{k}(x) d x=\sum_{\mu, \rho \in \mathbf{Z}_{+}^{N}} A_{\mu, \rho}\left\langle f, h_{\mu}\right\rangle \overline{\left\langle f, h_{\rho}\right\rangle},
$$

where

$$
A_{\mu, \rho}=\left(1+i^{|\rho|-|\mu|}\right) \cdot \sum_{j=1}^{N} \sum_{\nu \in I_{\mu} \cap I_{\rho}} c_{\nu, \mu}^{j} c_{\nu, \rho}^{j} .
$$

On the other hand, a short calculation, using formulas (3.2) and (4.1), shows that

$$
\left(|x|^{2}-\Delta_{k}\right) h_{\nu}=\sum_{j=1}^{N} \sum_{\mu \in I_{\nu}} \sum_{\rho \in I_{\mu}} c_{\nu, \mu}^{j} c_{\mu, \rho}^{j}\left(1+i^{|\rho|-|\nu|}\right) h_{\rho}=\sum_{\rho \in \mathbb{Z}_{+}^{N}} A_{\nu, \rho} h_{\rho}
$$

where for the last identity, we used the fact that the coefficients $c_{\nu, \mu}^{j}$ are symmetric in their subscripts: $c_{\nu, \mu}^{j}=\int_{\mathbb{R}^{N}} x_{j} h_{\nu}(x) h_{\mu}(x) w_{k}(x) d x=c_{\mu, \nu}^{j}$. But by Lemma 3.1.(3), the left side of $(4.3)$ is equal to $(2|\nu|+2 \gamma+N) h_{\nu}$. The linear independence of the $h_{\nu}$ now implies that

$$
A_{\nu, \rho}= \begin{cases}0 & \text { if } \rho \neq \nu \\ 2|\nu|+2 \gamma+N & \text { if } \rho=\nu\end{cases}
$$

Together with (4.2), this yields the assertion.

In view of Lemma 3.1.(1), and as $h_{0}$ is a constant multiple of $e^{-|x|^{2} / 2}$, we obtain as an immediate consequence the following:

Corollary 4.2. For $f \in L^{2}\left(\mathbb{R}^{N}, w_{k}(x) d x\right)$,

$$
\int_{\mathbf{R}^{N}}|x|^{2}\left(|f(x)|^{2}+\left|\hat{f}^{k}(x)\right|^{2}\right) w_{k}(x) d x \geqslant(2 \gamma+N) \cdot\|f\|_{2, w_{k}}^{2} .
$$

Moreover, equality holds if and only if $f(x)=c e^{-|x|^{2} / 2}$ with some constant $c \in \mathbb{C}$.

Proof of Theorem 1.1 We may assume that $\|f\|_{2, w_{k}}=1$. For $s>0$ define $f_{s}(x):=s^{-\gamma-N / 2} f(x / s)$. Since $w_{k}$ is homogeneous we easily see that

$\left\|f_{s}\right\|_{2, w_{k}}=1 \quad$ and $\quad \hat{f}_{s}^{k}(\xi)=s^{\gamma+N / 2} \cdot \hat{f}^{k}(s \xi) \quad$ for all $s>0$ and $\xi \in \mathbb{R}^{N}$. 
The above corollary implies that

$$
\Phi_{f}(s):=\int_{\mathbf{R}^{N}}|x|^{2}\left(\left|f_{s}(x)\right|^{2}+\left|\widehat{f}_{s}^{k}(x)\right|^{2}\right) w_{k}(x) d x \geqslant 2 \gamma+N
$$

On the other hand, we can write

$$
\Phi_{f}(s)=s^{2} \cdot\||x| f\|_{2, w_{k}}^{2}+\frac{1}{s^{2}} \cdot\left\||x| \widehat{f}^{k}\right\|_{2, w_{k}}^{2} .
$$

It is easily checked that $s \mapsto \Phi_{f}(s)$ takes a minimum on $(0, \infty)$, namely

$$
2 \cdot\||x| f\|_{2, w_{k}} \cdot\left\||x| \widehat{f}^{k}\right\|_{2, w_{k}}
$$

This implies (1.1). Further, equality in (1.1) holds exactly if $\min _{s \in(0, \infty)} \Phi_{f}(s)=2 \gamma+N$. By the second part of the corollary, this condition is satisfied if and only if $f(x)=c e^{-s^{2}|x|^{2} / 2}$ with some constants $c \in \mathbb{C}$ and $s>0$. This finishes the proof.

\section{REFERENCES}

[1] T.H. Baker and P.J. Forrester, 'Non-symmetric Jack polynomials and integral kernels', Duke Math. J. 95 (1998), 1-50.

[2] N.G de Bruijn, 'Uncertainty principles in Fourier analysis', in Inequalities, (O. Shisha, Editor) (Academic Press, New York, 1967), pp. 57-71.

[3] T.S. Chihara, An introduction to orthogonal polynomials, Mathematics and its Applications (Gordon and Breach, New York, 1978).

[4] D.L. Donoho and P.B. Stark, 'Uncertainty principle and signal recovery', SIAM J. Appl. Math. 49 (1989), 906-931.

[5] C.F. Dunkl, 'Differential-difference operators associated to reflection groups', Trans. Amer. Math. Soc. 311 (1989), 167-183.

[6] C.F. Dunkl, 'Integral kernels with reflection group invariance', Canad. J. Math. 43 (1991), 1213-1227.

[7] M.F.E. de Jeu, 'The Dunkl transform.', Invent. Math. 113 (1993), 147-162.

[8] M.F.E. de Jeu, 'An uncertainty principle for integral operators', J. Funct. Anal. 122 (1994), 247-253.

[9] L. Lapointe and L. Vinet, 'Exact operator solution of the Calogero-Sutherland model', Comm. Math. Phys. 178 (1996), 425-452.

[10] E.M. Opdam, 'Dunkl operators, Bessel functions and the discriminant of a finite Coxeter group', Compositio Math. 85 (1993), 333-373.

[11] A.P. Polychronakos, 'Exchange operator formalism for integrable systems of particles', Phys. Rev. Lett. 69 (1992), 703-705.

[12] M. Rösler, 'Generalized Hermite polynomials and the heat equation for Dunkl operators', Comm. Math. Phys. 192 (1998), 519-542.

[13] M. Rösler, 'Positivity of Dunkl's intertwining operator', Duke Math. J. (to appear).

[14] M. Rösler and M. Voit, 'An uncertainty principle for Hankel transforms', Proc. Amer. Math. Soc. 127 (1999), 183-194. 
[15] C.T. Roosenraad, Inequalities with orthogonal polynomials, thesis (Univ. of Wisconsin, 1969).

Zentrum Mathematik

Technische Universität München

Arcisstr. 21

D-80290 München

Germany

e-mail: roesler@mathematik.tu-muenchen.de 